\title{
Chronic pelvic pain
}

\author{
Talat Uppal', C Amarasekara² and Vasundhara Kaushik ${ }^{3}$
}

1,2Northern Beaches Maternity Service, Manly Hospital, Manly New South Wales 1655, Australia.

${ }^{3}$ Tara Imaging for Women, Campbelltown, New South Wales 2515, Australia.

Correspondence to author via ASUM.Email authors@asum.com.au.

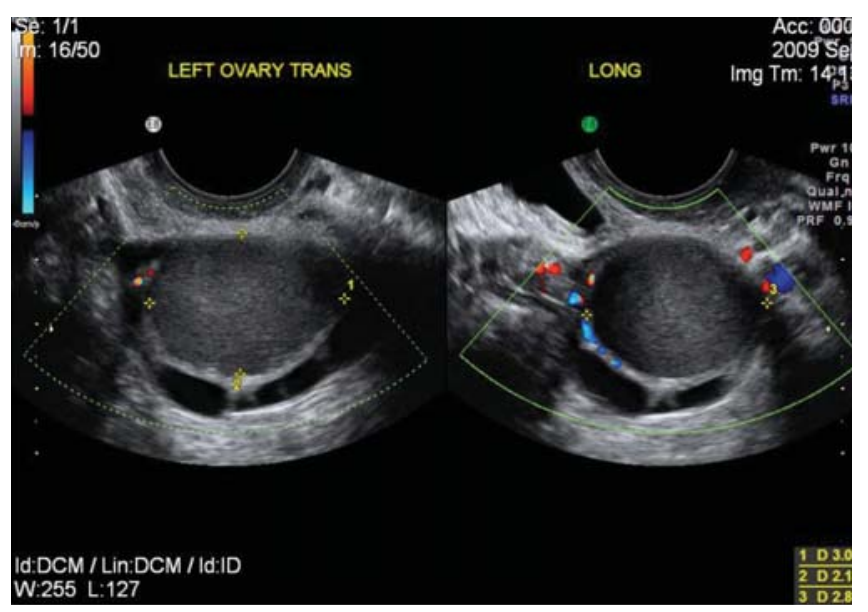

Fig. 1: Homogeneous low-level echoes with minimal peripheral vascularity.

\section{Definition}

Chronic Pelvic Pain (CPP) is defined as intermittent or constant pain in the lower abdomen or pelvis of at least six months duration, not occurring exclusively with menstruation or intercourse and not associated with pregnancy ${ }^{1}$.

Chronic Pelvic Pain is a symptom, not a disease, and can result from many different entities. As there is a significant socio-economic impact, it is important to triage, diagnose and manage these patients efficiently and accurately ${ }^{1}$.

\section{Epidemiology and management}

Chronic Pelvic Pain is a common ailment affecting up to one in six of the adult female population ${ }^{2,3}$.

Over $10 \%$ of all referrals to gynaecologists are for pelvic pain ${ }^{3}$.

The aetiology of chronic pelvic pain is complex and often multifactorial. The most common gynaecological causes of CPP are endometriosis and chronic pelvic inflammatory disease (PID). The non-gynaecological causes include musculoskeletal, urinary and gastrointestinal disorders.

Patients with CPP may have sexual, bladder or bowel dysfunction as well as generalised symptoms. The history should include checking for any coexistent anxiety, depression, somatoform disorders and/or drug addiction. There is often a combined psychological and physical contribution to the reduced quality of life $\mathrm{e}^{4-7}$.

A multidisciplinary approach to this clinical challenge is the best way forward and if the clinical presentation suggests a non-gynaecological component to CPP, referral to the relevant healthcare professional should be organised ${ }^{1,5,6}$.

The management team may include (depending on the symptoms and examination findings) a gastroenterologist, urologist, genitourinary medicine physician, physiotherapist, psychologist, social worker and a psychosexual counsellor ${ }^{1,4}$.

\section{Gynaecological causes of CPP \\ - Endometriosis}

- Adhesions (chronic pelvic inflammatory disease/ previous pelvic surgery)

Uterine fibroids

- Ovarian cysts/dermoids

- Pelvic congestion syndrome

- Adenomyosis

- Malignancy.

\section{Non-gynaecological causes of CPP}

Irritable bowel syndrome/diverticular disease

- Bladder hypersensitivity

- Nerve entrapment

Musculoskeletal basis.

\section{The role of pelvic ultrasound}

This non-invasive diagnostic tool has a significant place in the investigation of patients with chronic pelvic pain.

It is commonly used to help identify pelvic masses or cysts and their origin, uterine adenomyosis, prominent pelvic varicosities, and hernias (spigelian hernias).

We have reviewed four cases which highlight different presentations of CPP.

\section{Case 1}

This was a 26-year-old primiparous woman who presented with a ten-month history of right pelvic pain. She was considering pregnancy in the near future.

The pelvic ultrasound revealed a normal sized uterus and right ovary.

The left ovary however was expanded by a thin walled cystic structure measuring about $10 \mathrm{~mL}$ in volume and filled with homogeneous low-level echoes with minimal peripheral vascularity (see Fig. 1). These are typical ultrasound features of an endometrioma.

The classic unilocular ground glass appearance of an endometrioma results from multiple bleeding episodes and tiny reorganising clots of low/medium echo. The cyst tends to become thick walled over time representing a fibrotic wall containing both new and old blood in the lumen. They are often bilateral.

Endometriomas have other less common ultrasonographic presentations. These include a complex cyst with septations containing both solid and cystic components. Nodularity may occur, typically associated with normal impedance blood flow on spectral Doppler interrogation ${ }^{8.9}$.

The cyst can also appear as a haemorrhagic cyst, the latter usually having internal echoes in reticular pattern ${ }^{8.12}$. The definitive diagnosis of an endometrioma is made on histopathological grounds. Up to 50 per cent of women with endometriosis develop endometriomas. 


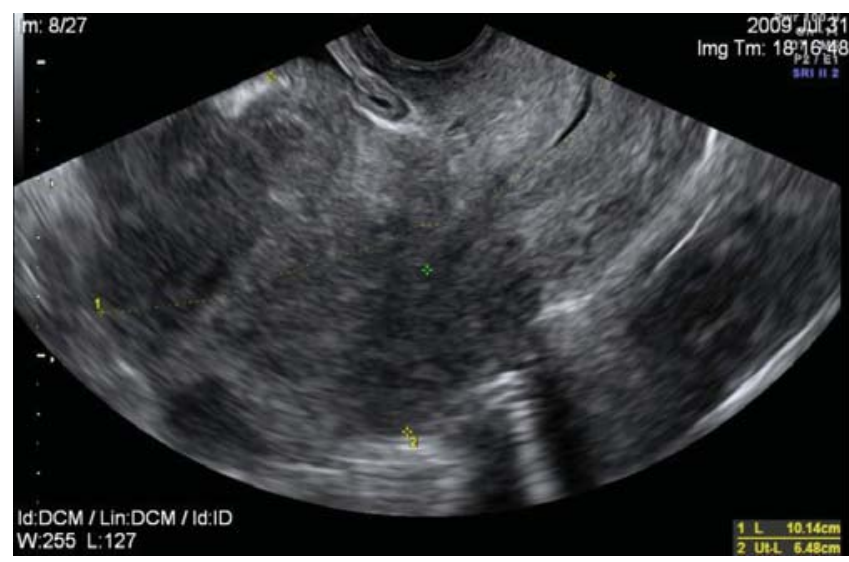

Fig. 2: Postero/fundal aspect of the uterus.

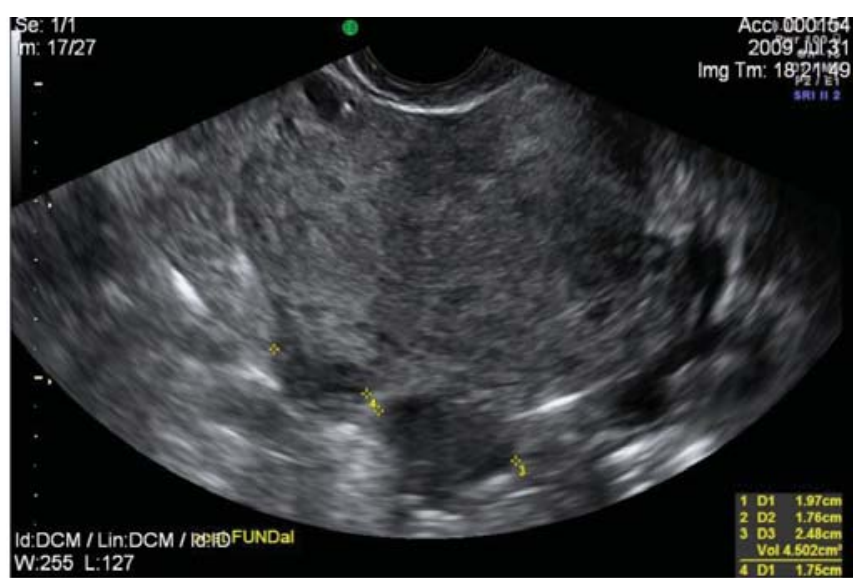

Fig. 3: Postero/fundal aspect of the uterus..

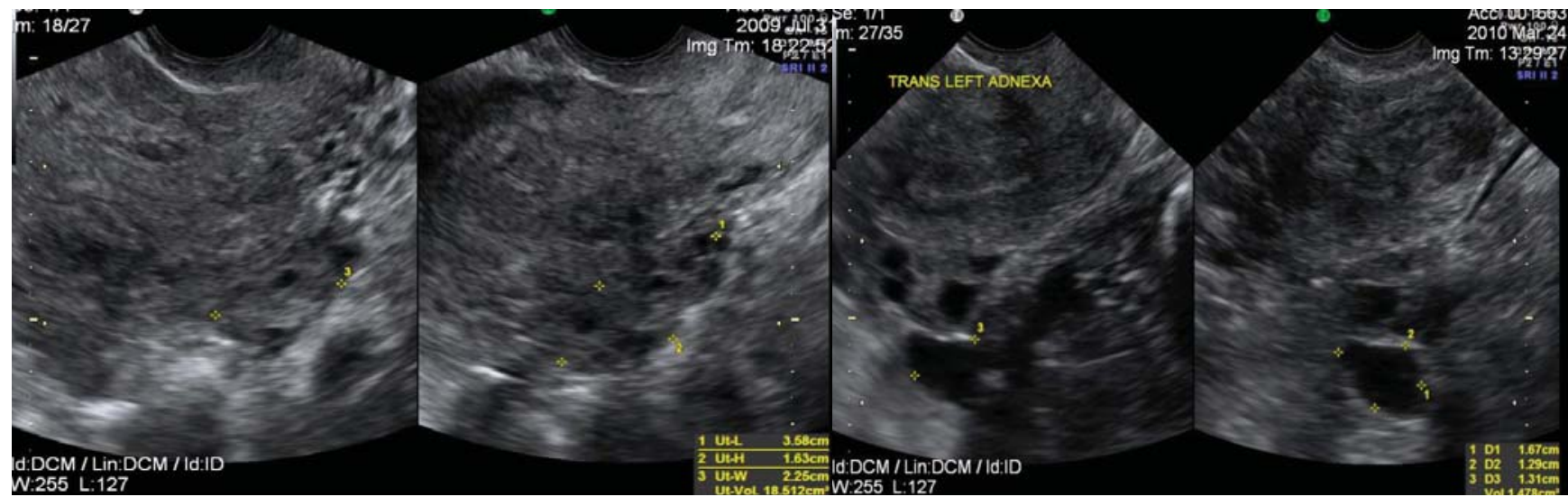

Fig. 4: A retroverted uterus with frozen non-mobile ovaries.

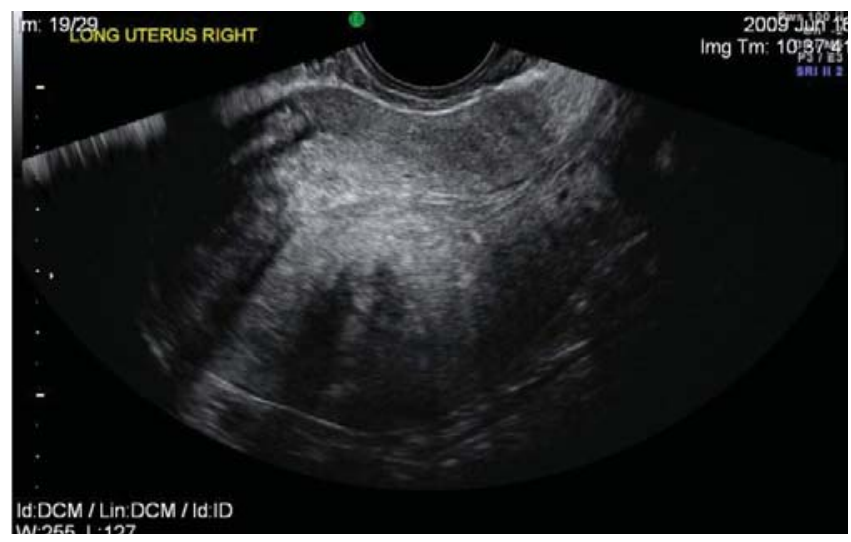

Fig. 5: The myometrium.

\section{Case 2}

This was a 41-year-old nulliparous woman with past history of symptomatic endometriosis (dyschezia/dyspareunia) involving a rectosigmoid bowel which was adherent to the posterior aspect of the uterus. She subsequently had extensive surgery with bowel re-anastomosis three years prior to presentation. She was medicated (norethisterone) to improve her symptoms, which had improved post operatively but persisted to a milder degree.

The pelvic ultrasound findings included hypoechoic structures contiguous with the postero/fundal aspect of the uterus (Figs. 2 and 3) which were distinctly tender to transducer pressure which favoured them to be endometrial deposits rather than fibroids.

There was also exquisite tenderness to transducer pressure on palpation of both ovaries. Both ovaries and the uterus demonstrated minimal mobility. The findings were suggestive of active disease and periovarian adhesions secondary to the endometriosis and/or surgery.

Diffuse endometriosis can be difficult to pick on ultrasound and needs a high index of clinical suspicion to diagnose this condition.

There may be tiny deposits along the posterior aspect of the uterus with hypoechoic centers which can mimic the bowel. The former are, however, fixed in position, do not change shape, do not demonstrate acoustic streaming and are usually tender to transducer pressure ${ }^{8,12}$.

The assessment of mobility and tenderness including location of the tenderness is of utmost importance to the diagnosis however may be overlooked ${ }^{11}$.

A retroverted uterus with frozen non-mobile ovaries (Fig. 4) due to pelvic adhesions on transducer palpation are all clues towards the likely diagnosis.

As the bladder fills up with a small amount of urine it is easy to assess bladder wall at the conclusion of a transvaginal study for endometriomas ${ }^{8,12}$.

\section{Case 3}

This was a 50-year-old G6 P4 lady who presented with painful, heavy periods. She had been hormonally medicated to minimise the bleeding.

The pelvic ultrasound demonstrated typical features of adenomyosis including the bulky globular uterus with heterogenous appearance of the myometrium (Fig. 5). There was asymmetrical enlargement of the posterior myometrium with shadowing in Venetian blind pattern (Figs. 5 and 6). The endometrial/myometrial interface was difficult to define. The uterus was generally tender to transducer pressure with increased vascularity (Fig. 7). 


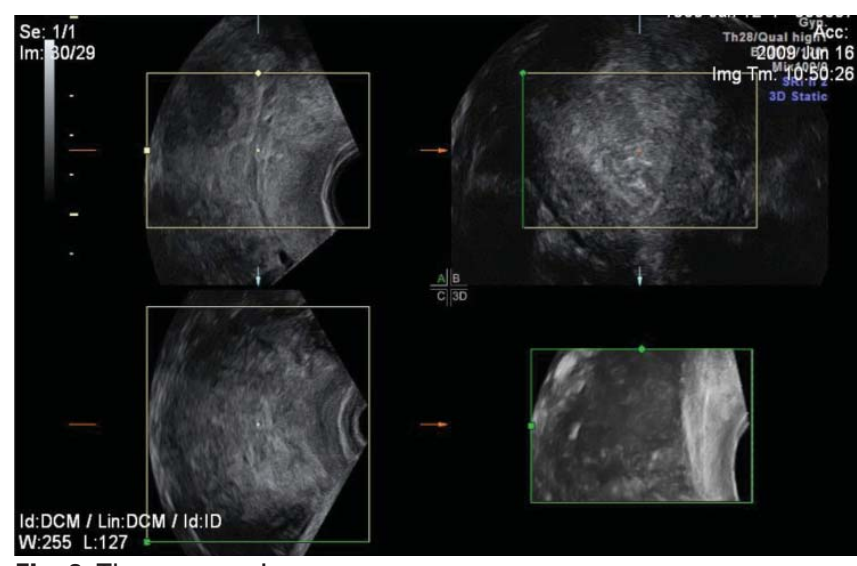

Fig. 6: The myometrium.

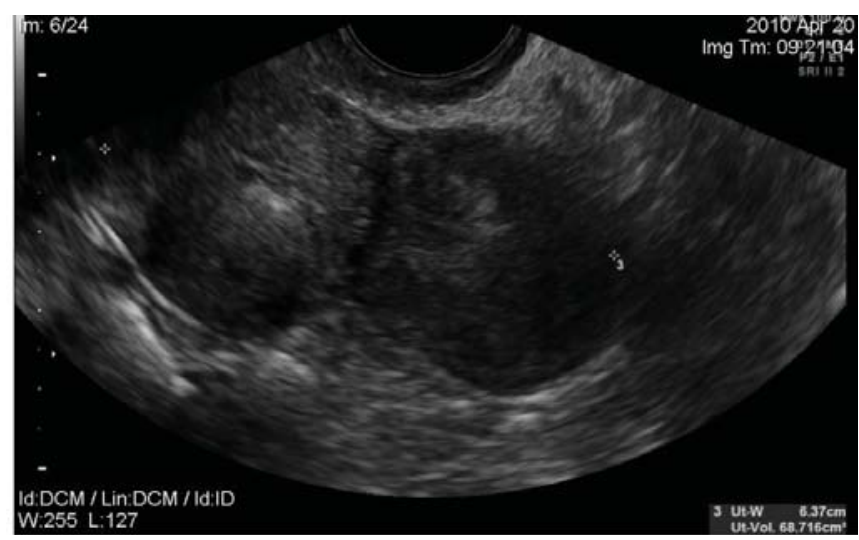

Fig. 8: Right lateral/fundal pedunculated fibroid.

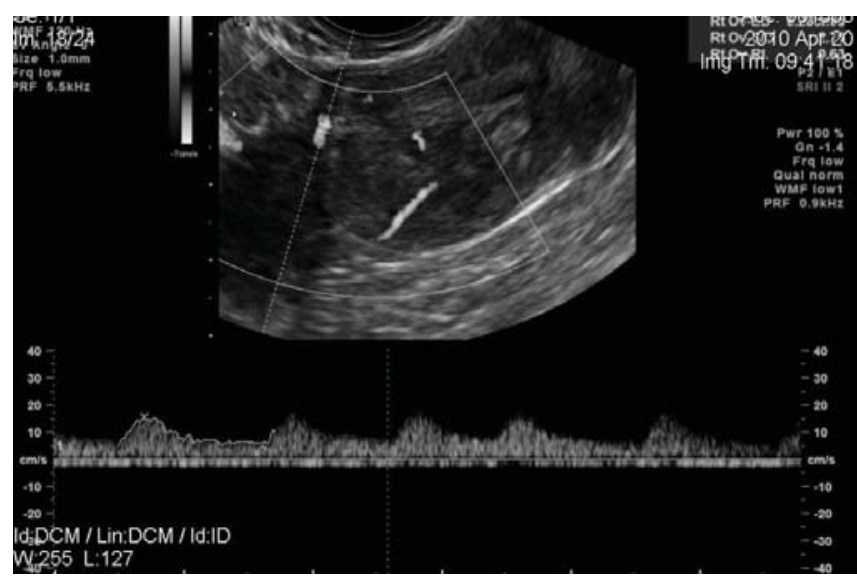

Fig. 10: Pulse Doppler interrogation of pedicle.

Adenomyosis is the presence of endometrial glands and stroma within the uterine musculature. Symptoms typically occur in women between 40 and 50 years of age, this pathology is more common among multiparous than in nulliparous women.

The ectopic endometrial tissue causes myometrial hypertrophy producing a diffusely enlarged and globular uterus with posterior shadowing in a venetian blind pattern on ultrasound. Tiny intramyometrial cysts may be visible. These ultrasound features have high sensitivity and specificity for adenomyosis although tissue analysis is the diagnostic gold standard ${ }^{7,11}$.

\section{Case 4}

This was a 61-year-old P0 woman who presented with worsening lower abdominal discomfort for six months on a background of active diverticular disease.

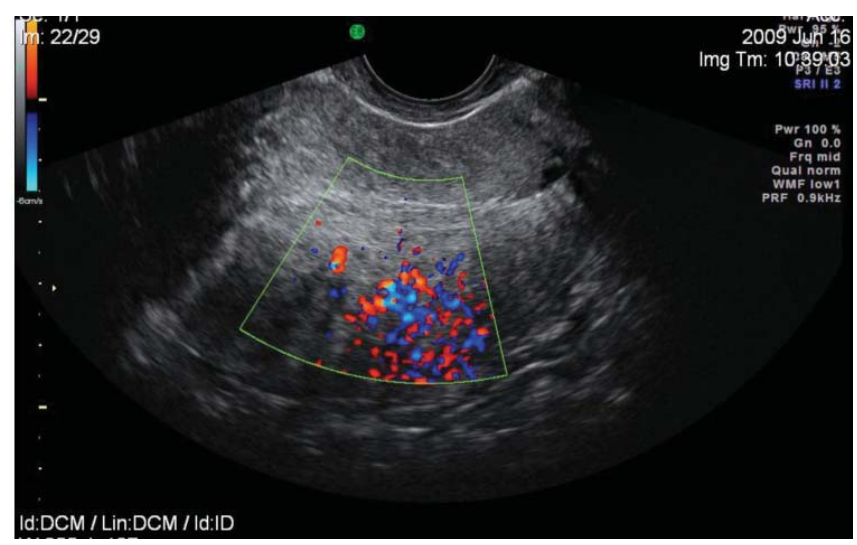

Fig. 7: The uterus was generally tender to transducer pressure with increased vascularity.

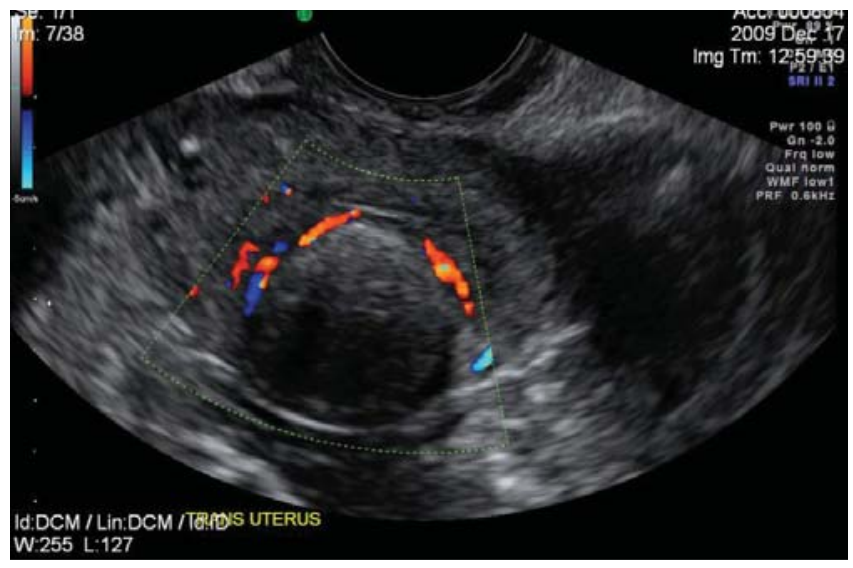

Fig. 9: Doppler trace of vascular pedicle.

Pelvic ultrasound demonstrated multiple intramural fibroids. The anterofundal fibroid displaced the endometrium posteriorly. It was difficult to exclude a submucosal component.

Two right solid appearing, ovoid, heterogeneous masses were also demonstrated, the larger of which appeared to be attached to the uterine fundus with a broad vascular pedicle (Figs. 8, 9, 10).

The left ovary was separate to the adnexal mass and inferior to it. The findings were suggestive of pedunculated and broad ligament fibroids.

This was a patient with gynaecological and non gynaecological aetiologies for her CPP, managed by her GP, gynaecologist and physician.

The case also highlighted that unless a clear pedicle is seen arising from the uterus it can be difficult to distinguish between a malignant mass and vascular fibroids in a postmenopausal woman ${ }^{8,10}$.

The patient elected to have a hysterectomy and leiomyomata were confirmed on histopathology.

\section{Pelvic congestion syndrome}

Pelvic venous syndromes are disorders of the pelvic venous circulations, and include vulvovaginal varicosities (VVV) and pelvic congestion syndrome (PCS). They represent a controversial topic of uncertain aetiology compounded by the current lack of definitive diagnostic criteria. Tuboovarian varicoceles are considered the female equivalent of testicular varicocele ${ }^{13-18}$.

Epidemiologically, roughly $4 \%$ of women suffer from VVV. PCS is most prevalent in multiparous women in the reproductive age group. It is possible that this reflects the 
$50 \%$ increase in pelvic vein capacity during pregnancy which may lead to venous incompetence and reflux in the non-pregnant state. Indeed PCS is implicated in up to a third of CPP patients in whom no other obvious pathology can be found $^{13,15,18,19}$.

1 VVV occur often in pregnancy and the tendency is for the varices to resolve spontaneously after delivery. They are thought to be secondary to raised venous pressure and venous insufficiency. The patient may have VVV as a separate entity or may have lower limb varices in addition. VVV may also occur as a component of PCS.

2 Pelvic congestion syndrome is typically characterised by chronic pelvic discomfort or pain in women of minimum six months duration, with periovarian varicosities on imaging studies. The CPP or discomfort is usually described as an awareness of unilateral pelvic heaviness or a dull pain worsened premenstrually, or by standing for long periods and/or sexual intercourse. Accordingly, the diagnosis of PCS is based on a history of such symptoms, tenderness on internal examination, and pelvic vein dilatation or incompetence on imaging/ laparoscopy. Other causes for these non specific findings need to be excluded ${ }^{15,17,18}$.

It is postulated that hormonal or gravitation/anatomic factors can cause venous insufficiency of the ovarian veins and/or internal iliac veins, leading to periovarian pelvic varicosities. On the other hand, ovarian vein dilatation and reflux on pelvic venography are common findings in asymptomatic multiparous premenopausal women.

Ultrasonography is the first line imaging study and is used in the diagnosis of PCS. This is basically to exclude pelvic neoplasms and uterine pathology as potential causes of pain.

\section{Ultrasonographic features which support PCS diagnoses}

- Dilatation of the left ovarian vein with reversed caudal flow

- Tortuous and dilated pelvic venous plexuses

- Polycystic changes of the ovary

- Dilated arcuate veins crossing the uterine myometrium

Variable duplex waveform in varicoceles during the Valsalva manoeuvre ${ }^{17,18,19}$.

Although the lack of pelvic venous changes aids in excluding PCS, the presence of these abnormalities is not diagnostic, as dilated and incompetent ovarian veins are common, nonspecific findings. There is a lack of agreement on the exact cut-off for ovarian vein diameter in PCS, and no validated measure for venous congestion or tortuosity ${ }^{16-18}$.

Sensitivity is higher if the ultrasonographic study is performed with the patient upright/doing valsalva manoeuvres. Even with these procedures the correlation between ultrasound findings and venography for the presence or absence of pelvic varices is poor ${ }^{13,18}$.

In general, computed tomography (CT) and magnetic resonance (MR) imaging may offer better detail of tortuous, dilated pelvic and ovarian veins, broad ligament vascular congestion and ovarian varicoceles.

\section{References}

1 Royal College of Obstetricians and Gynaecologists Greentop Guideline number 41: April 2005. Management of chronic pelvic pain.

2 Zondervan K, Barlow DH. Epidemiology of chronic pelvic pain. Best Pract Res Clin Obstet Gynaecol 2000; 14: 403-14.

3 Zondervan KT, Yudkin PL, Vessey MP, Jenkinson CP, Dawes MG, Barlow DH, Kennedy SH. The community prevalence of chronic pelvic pain in women and associated illnessbehaviour. Br J Gen Pract 2001; 51: 541-7.

4 Neis KJ, Neis F. Chronic pelvic pain: cause, diagnosis and therapy from a gynaecologist's and an endoscopist's point of view. Gynecol Endocrinol 2009; 25 (11): 757-61.

5 Weijenborg PT, Ter Kuile MM, Stones W. A cognitive behavioural based assessment of women with chronic pelvic pain. J Psychosom Obstet Gynaecol 2009; 30 (4): 262-8.

6 Howard FM. 2003. Chronic pelvic pain. Obstet Gynecol 101: 594-611.

7 Fall M, Baranowski AP, Elneil S, Engeler D, Hughes J, Messelink EJ, Oberpenning F, de C Williams AC; European Association of Urology. Guidelines on chronic pelvic pain. In European Association of Urology Guidelines, 2008 edn. Available online at: http://www. uroweb.org/files/uploaded_2326_files/guidelines/chronicpelvicpain. pdf [verified February 2011].

8 Meredith S, Sanchez-Ramos L, Kaunitz A. Diagnostic accuracy of transvaginal sonography for the diagnosis of adenomyosis:a systemic review and meta analysis. Am J Obstet Gynecol 2009; 201 (1): 107.

9 Heilbrun ME, Olpin J, Shaaban A. Imaging of benign adnexal masses: characteristic presentations on ultrasound, computed tomography, and magnetic resonance imaging. Clin Obstet Gynecol 2009; 52 (1): 21-39.

10 Hamper UM, Sheth S, Abbas FM, Rosenshein NB, Aronson D, Kurman RJ. Transvaginal color Doppler sonography of adnexal masses: differences in blood flow impedance in benign and malignant lesions. Am J Roentgenol 1993; 160 (6): 1225-8.

11 Fasih N, Shanbhogue AKP, Macdonald DB, Fraser-Hill MA, Papadatos D, Kielar AZ, Doherty GP, Walsh C, McInnes M, Atri M. Leiomyomas beyond the uterus: unusual locations, rare manifestations. Radiographics 2008; 28 (7); 1931-48.

12 Margit dueholm and Erik Lundorf. Transvaginal ultrasound or MRI for diagnosis of adenomyosis. Curr Opin Obstet Gynecol 2001; 18: 505-12.

13 Piketty M, Choplin N, Bertrand Dosset, Millischer-bellaische A, Gilles R, Leconte M, Borghese B, Chapron C. Preoperative workup for pateints with deeply infiltrating endometriosis: transvaginal ultrasnography must definitely be the first-line imaging examination. Human Reprod 2009; 24: 602-7.

14 Hobbs, JT. The pelvic congestion syndrome. Br J Hosp Med 1990; 43: 200 .

15 Wang, S, Lang, JH, Zhou, HM. Venous malformations of the female lower genital tract. Eur J Obstet Gynecol Reprod Biol 2009; 145: 205.

16 Fassiadis, N. Treatment for pelvic congestion syndrome causing pelvic and vulvar varices. Int Angiol 2006; 25:1.

17 Tu FF, Hahn D, Steege JF. Pelvic congestion syndrome-associated pelvic pain: a systematic review of diagnosis and management. Obstet Gynecol Surv 2010; 65: 332.

18 Tropeano, G, Di Stasi, C, Amoroso, S, et al. Ovarian vein incompetence: a potential cause of chronic pelvic pain in women. Eur J Obstet Gynecol Reprod Biol 2008; 139: 215.

19 Johnson N. Vulvovaginal varicosities and pelvic congestion syndrome. Available online at: http://www.uptodate.com [verified September 2009]. 